\title{
Confluences et renaissance dans Highland River (1937) ? Chronotope, allégorie et idéologie
}

Confluence and Rebirth in Highland River (1937)? Chronotope, Allegory, and Ideology

Philippe Laplace

\section{(2) OpenEdition}

Journals

Édition électronique

URL : http://journals.openedition.org/etudesecossaises/1298

ISSN : 1969-6337

Éditeur

UGA Éditions/Université Grenoble Alpes

Édition imprimée

ISBN : 978-2-37747-001-3

ISSN : $1240-1439$

Référence électronique

Philippe Laplace, « Confluences et renaissance dans Highland River (1937) ? Chronotope, allégorie et idéologie », Études écossaises [En ligne], 19| 2017, mis en ligne le 01 avril 2017, consulté le 08 septembre 2020. URL : http://journals.openedition.org/etudesecossaises/1298

Ce document a été généré automatiquement le 8 septembre 2020.

(c) Études écossaises 


\title{
Confluences et renaissance dans Highland River (1937) ? Chronotope, allégorie et idéologie
}

\author{
Confluence and Rebirth in Highland River (1937)? Chronotope, Allegory, and \\ Ideology
}

Philippe Laplace

1 La confluence, que l'on peut définir comme la convergence vers un même lieu de plusieurs forces, soient-elles physiques ou intellectuelles, ou de plusieurs courants, est un concept qu'illustre à merveille le roman que Gunn publia en 1937 et qui lui donna assez d'assurance et de prestige pour se lancer comme écrivain professionnel. Célébré pour sa puissance évocatrice, Highland River n'en demeure pas moins un roman complexe par sa forme et à cause de la trame et des éléments narratifs choisis par Gunn. Un des facteurs de complexité et de confusion de l'œuvre réside aussi dans le fait que le roman appartient à plusieurs genres littéraires. On peut d'abord le considérer comme un Bildungsroman, c'est-à-dire un roman d'apprentissage, avec un protagoniste que l'on suit depuis sa tendre enfance dans son village des Hautes-Terres, puis durant la Première Guerre mondiale qu'il passe sur le front en France et jusqu'à la remontée qu'il entreprend vers la source de sa rivière des Hautes-Terres, un véritable pèlerinage. On peut aussi considérer ce roman comme un roman initiatique, avec des phases, des épreuves et des archétypes qui rappellent la structure classique des contes de fées (Laplace, 2004). Le chemin du protagoniste est en effet parsemé d'adjuvants et d'opposants qui l'orientent et le guident vers son but ultime, la source (Greimas, 1970). Il ne faut certes pas non plus écarter la perspective régionaliste : ce roman, comme presque tous ceux de Gunn, est localisé dans un cadre spécifique bien particulier, celui de son Caithness natal. Malgré quelques scènes à Glasgow ou sur le Front de la Somme, par exemple, il est difficile de ne pas catégoriser Highland River autrement qu'avec l'adjectif régionaliste, un terme qui n'est d'ailleurs pas nécessairement péjoratif et que Gunn n'était de toute façon pas sans revendiquer pour son œuvre: «If my work could be regarded as in any way a tribute to the Highlands and its people, I should be pleased " avait-il 
par exemple déclaré (1961). Mais ces genres littéraires peuvent être combinés, pour reprendre la thématique de la confluence, en ce que Bakhtine nomma chronotope, c'est-à-dire une structure qui regroupe les caractéristiques spatio-temporelles d'un récit. Nous verrons comment Gunn a eu recours à ce procédé littéraire dans Highland River pour formuler ses réflexions sur la politique écossaise et ses points de vue idéologiques.

Cet article va d'abord étudier ce roman sous un angle bakhtinien avant d'examiner les ambitions idéologiques que Gunn tenait à exprimer grâce à ces éléments spatiotemporels. L'objectif est en effet montrer que le romancier était parvenu à échafauder un projet allégorique avec Highland River, dessein qui a jusque-là été totalement passé sous silence par la critique gunnienne, trop occupée à démontrer comment ce roman marque le début de sa réflexion philosophique et les premiers éléments d'une pensée tournée vers l'Orient.

3 Les théories littéraires bakhtinienne apparaissent pertinentes pour les romans de Gunn, comme par exemple les principes d'hétéroglossie - c'est-à-dire les différentes voix, y compris celle de l'auteur, qui composent un roman - et par extension de monoglossie, un trait dont on peut parfois accuser Gunn. Le sont également les analyses de Bakhtine sur les Bildungsroman et les romans régionalistes qu'il considérait comme le prolongement de ce qu'il nommait des « romans idylles». Selon Bakhtine, il ne fallait par exemple pas négliger les romans régionalistes, car ils sont fondamentaux à l'expression de toute une palette idéologique (1978, p. 371), et c'est justement un point pertinent à la lecture de Highland River proposée dans cet article. C'est cependant au niveau des chronotopes que ce roman est le plus représentatif de la pensée du critique russe : ce concept livre des clés permettant d'expliciter les rapports étroits que Gunn envisageait entre les personnages, les Hautes-Terres et l'histoire, des liens intrinsèques qui sous-tendent la plupart ses romans. Le terme de chronotope doit être entendu comme «la corrélation essentielle des rapports spatio-temporels, telle qu'elle a été assimilée par la littérature. [...] Ce qui compte pour nous c'est qu'il exprime l'indissolubilité de l'espace et du temps (celui-ci comme quatrième dimension de l'espace) » (ibid., p. 237). Il ne s'agit donc pas d'un genre particulier mais d'un cadre qui régit les caractéristiques narratives d'un récit, dans lequel les premières s'expriment. Pour Bakhtine, le chronotope doit d'abord être envisagé comme force créatrice et pas comme simple unité géographique et temporelle d'un récit. Le chronotope est un moteur: c'est l'interaction entre topos et chronos, dans laquelle se trouve le protagoniste, qui donne le point de départ et lance l'intrigue, par exemple dans des romans historiques, ou qui permet à l'intrigue de se développer, comme pour des romans d'aventure (1986, p. 49).

4 L'intrigue de Highland River s'inscrit parfaitement dans cette optique. Au fur et à mesure de ses pérégrinations le long de la rivière, qui commencent comme des expéditions de braconnage avant de se transformer en une quête personnelle, Kenn, le protagoniste que Gunn hisse déjà à un statut très symbolique grâce à son prénom, découvre les ruines des civilisations passées. Depuis les Pictes jusqu'aux vestiges de maisons abandonnées lors des évictions, chaque communauté - Gunn emploie le terme polémique et maladroit de « race » - a laissé des traces de son passage sur les berges de la rivière. Mais cette exploration révèle surtout aussi à Kenn des éléments de sa personnalité, de sa culture et de son identité. Espace et temps sont donc intimement liés dans la trame narrative du roman et fournissent le cadre dans lequel protagoniste 
et récit évoluent. Pour le jeune Kenn, l'histoire qu'il apprend sur les bancs de l'école, celle qui se passe dans les capitales européennes et qui concerne des gens et des lieux qu'il ne connaît pas, n'est que dénaturée et stérile puisque coupée des réalités pragmatiques de son monde, de son habitat. Ses ancêtres pictes ou vikings ne sont pas reconnus comme pertinents au grand récit de l'histoire tel qu'il lui est conté, ils ne sont que : "[...] sounds in the empty spaces of history " (Gunn, 1937, p. 53) alors que le maître insiste sur la nécessité de bien connaître les principales villes anglaises et leurs industries. Mais pour Kenn, seules les connaissances liées à l'histoire de sa région et de sa communauté peuvent combler ce vide et donner un sens à son existence.

5 La confluence des données spatiales et temporelles pour un protagoniste est bien sûr omniprésente dans les romans de Gunn: son territoire, les Hautes-Terres, est intimement associé à des événements qui ont un ancrage précis dans le temps, que ce soit les invasions pictes, les évictions ou le renouveau économique des régions côtières des Hautes-Terres. Mais cette confluence est aussi présente dans des romans dont l'intrigue n'est pas à proprement parler liée à l'histoire, tout comme Highland River, mais où celle-ci apparaît en filigrane.

6 Bakhtine avait de plus insisté sur le fait que le lieu est inséparable des personnages qui y évoluent, élément essentiel pour la caractérisation des personnages de Gunn. Espace et temps s'inscrivent donc dans un cadre ontologique, d'abord celui de l'identité puis celui du devenir du protagoniste. Cette dimension et ce cadre sont bien sûr eux aussi intrinsèques aux romans de Gunn où, à de rares exceptions, les protagonistes ne font qu'un avec leur communauté, leurs traditions, l'espace et le temps : "This immemorial heritage has become part of our unconscious self ", déclara Gunn dans un article critique (1929, p. 335) et dans Highland River la rivière acquiert une dimension mnésique ; Kenn, en remontant jusqu'à la source, retrouve les traces du passé de sa civilisation. Mais les romans de Gunn reflètent aussi sa vision, parfois naïve, de la politique, de l'économie et de la société. Gunn se servait de ses récits comme d'une plateforme à partir de laquelle il exprimait ses espoirs nationalistes. En 1952, dans un article inédit destiné à une agence de tourisme, il écrivit par exemple, utilisant la première personne du pluriel afin de se faire la voix des habitants des Hautes-Terres : "We like to contemplate a kind of community where the ancient values of Highland culture (including our attitude to time) may be perpetrated amid an economy which will give a good life for all. We think it can be done." (1952, p. 28)

7 Si le chronotope est une dimension fondamentale de ses récits, il faut quand même admettre que Gunn reste très souvent figé dans un cadre nostalgique, mis à part bien entendu lors de ses récits sur les évictions, même si son écriture est fortement empreinte de mélancolie. Dans beaucoup de ses écrits, c'est le fabuleux âge d'or des communautés gaéliques qui est mis en avant et les narrateurs ne peuvent que se lamenter de sa disparition. Gunn reconnaissait après tout, bon gré mal gré, la fascination naturelle qu'exerçait le Crépuscule celtique pour un écrivain des HautesTerres ainsi que l'attrait de la notion d'un âge d'or gaélique (1987, p. 102 ; voir aussi Hart \& Pick, 1985, p. 96-97). En dépit des prises de position du protagoniste de Highland River qui se défend de toute conception nostalgique du passé, il est difficile de ne pas considérer ainsi ses réflexions sur les populations qui ont habité les berges de sa rivière. À un collègue de travail qui l'accuse précisément de faire montre de trop de nostalgie, Kenn répond que le terme Crépuscule celtique émane d'un jugement et d'une vision extérieurs à la communauté : 
I never had any experience of this Twilight. An old woman in the old days in my country knew the nature of what grew around her quite precisely. She gathered her roots and her lichens and out of them made the vivid dyes you see in tartans. That was what happened in fact. It is clear too, that they achieved in their way a very tolerable communal life, and worked to the rhythm of their own music and that sort of thing. (1937, p. 215)

Cette tentative de réfutation correspond clairement aux généralisations que Gunn avait à maintes fois utilisées contre ceux qui l'accusaient de propager une vision nostalgique des Hautes-Terres. Seuls ceux qui appartiennent à ces communautés peuvent les comprendre ; les autres, comme le collègue de Kenn originaire de Pologne, ne peuvent pas concevoir la vie et la culture des Hautes-Terres. Ils en sont réduits à utiliser des clichés et affublent dédaigneusement de l'étiquette «Crépuscule celtique » toutes ces conceptions. Gunn avait aussi à cœur de se défendre en détournant la raison d'être de ce qui était selon lui trop aisément appelé « Crépuscule » : au lieu d'être considéré dans sa dimension mélancolique, le passé devait être envisagé comme un vivier à partir duquel les individus et communautés pouvaient se ressourcer, pouvaient retrouver leur identité, exactement comme Kenn qui, dans son roman, exprime cette nécessité et ce frisson qui le font remonter jusqu'à la source de sa rivière des Hautes-Terres: "This journey, tentative at first, [...] became in the end a thrilling exploration into the source of the river and the source of himself. $(1937$, p. 52)

Gunn investit donc le chronotope bakhtinien d'une dimension rhizomatique : ce temps et ce lieu ont à plusieurs reprises, tout au long du roman, des prolongements et des répercussions sur les sensations qu'éprouvent Kenn ou ses parents, qu'elles soient psychologiques ou physiologiques. Le chronotope, ce syncrétisme entre le lieu et le temps historique, joue un rôle primordial chez Gunn sur l'état mental ou physique des personnages. Au fur et à mesure de sa remontée, Kenn perçoit des sensations qu'il ne peut exprimer autrement qu'avec des concepts métaphysiques : son environnement, et la connaissance qu'il acquiert de ce monde, ont des répercussions sur son bien-être et sa réactivité. Gunn déploie ici des concepts qu'il avait déjà exposés dans des articles à propos du rôle de l'environnement historico-social et de l'identité telle que l'appréhende le protagoniste $(1929,1932,1956)$. C'est aussi à partir de ces notions que l'on peut élever ce roman à une dimension métaphorique. Le symbolisme idéologique de Highland River prend alors toute sa signification, une interprétation jusque-là passée sous silence par la critique. Parmi la dizaine d'articles consacrés spécifiquement à Highland River aucun n'étudie en effet les remous qui agitaient les courants nationalistes et qui étaient contemporains à l'écriture du roman. Gunn avait pourtant confié que le climat politique de cette époque avait pesé sur son travail d'écrivain. Interrogé en octobre 1935 par Frank Morley, un des administrateurs de Faber \& Faber qui s'inquiétait de la progression de ce nouveau roman, Gunn avait imputé son retard à cette atmosphère pesante : "About your River letter. I'm doing nothing now but politics. Haven't even time to think of a place called the literary world. (Hart \& Pick, 1985, p. 138)

Choisir un cours d'eau pour exprimer des conceptions symboliques et idéologiques n'était certes pas un procédé littéraire nouveau. Le symbolisme de la rivière est prégnant dans toutes les littératures et à toutes les époques. Prenons par exemple un des romans les plus célèbres à propos de la remontée d'une rivière, Heart of Darkness. Conrad utilise le fleuve et son symbolisme dans un contexte archétypal supposé, beaucoup plus large que les divagations de Kenn qui ne touchent qu'à sa communauté. Les origines du monde sont évoquées par Conrad pour qui les berges du cours d'eau renferment des vestiges primitifs : 
Going up that river was like travelling back to the earliest beginnings of the world, when vegetation rioted on the earth and the big trees were kings. An empty stream, a great silence, an impenetrable forest. The air was warm, thick, heavy, sluggish. There was no joy in the brilliance of sunshine. The long stretches of the waterway ran on, deserted, into the gloom of overshadowed distances. [...] There were moments when one's past came back to one, as it will sometimes when you have not a moment to spare for yourself; but it came in the shape of unrestful and noisy dream, remembered with wonder amongst the overwhelming realities of this strange world of plants, and water and silence. And this stillness of life did not in the least resemble a peace. It was the stillness of an impalpable force brooding over an inscrutable intention. It looked at you with a vengeful aspect. (1899, p. 182-183)

11 Si le symbolisme de la rivière est menaçant chez Conrad et si la remontée du fleuve est une expérience angoissante pour Marlow, cette pérégrination est au contraire source de paix et de révélation mystique chez Gunn. La vision primitiviste de Conrad n'a pas sa place dans le discours traditionnaliste et idéologiquement marqué du romancier écossais. La palette symbolique que Gunn développe est limitée à un espace-temps celtique et préceltique et est empreint de concepts culturels et politiques écossais. Difficile en effet de ne pas considérer le chronotope dans Highland River comme une réflexion sur les espoirs littéraires et nationalistes caressés par Gunn. La rivière est le fil conducteur, le fil d'Ariane, qui permet au protagoniste de remonter le temps et de suivre, sans carte ni boussole, un chemin qui le mène, loin de tout parcours connu et balisé, droit vers le centre de son pays et de son identité, «[...] a stream running through time in a distant place » (Gunn, 1937, p. 185).

Il s'agit bien sûr d'une image très symbolique, associant temps et lieu à l'individualité, puisque Kenn est en fait sur le chemin de la découverte de soi, de la renaissance. Mais Gunn, en tissant cet écheveau, parvient échafauder une allégorie, c'est-à-dire un autre discours éloigné de son sens littéral et lui permettant d'exprimer ses préoccupations à propos de la situation des partis nationalistes et du climat agité sur les plans culturel et politique, comme en témoigne la grave crise de confiance que traversait le SNP à l'époque.

13 D'un point de vue politique, le SNP, créé depuis 1934, semblait être au plus bas en 1936 et 1937. Deux ans auparavant, lors de sa première présentation aux élections législatives en 1935, le SNP avait essuyé une très lourde défaite avec un résultat de 1,1 \%. La fusion du National Party of Scotland et du Scottish Party avait, contrairement à ce qui avait été envisagé, émoussé la dynamique nationaliste. Gunn qui, bien que resté en coulisse, avait été un des architectes de la fusion du Scottish Party et du National Party of Scotland (Finlay, 1994, p. 128), c'est-à-dire de cette confluence et de l'éclosion $\mathrm{du}$ nouveau parti politique, ne pouvait que se lamenter de cette situation. Celle-ci avait d'ailleurs mené le SNP à une crise idéologique majeure en 1937 : son secrétaire national, John MacCormick, s'était en effet résolu à chercher le soutien d'autres partis politiques favorables à une politique d'auto-détermination écossaise, faisant ainsi renaître de ces cendres le principe de la Scottish Home Rule Association et condamnant ainsi sans doute le parti politique à la disparition (Lynch, 2002, p.51). C'est ce dessein, auquel Gunn était fermement opposé, que MacCormick avait commencé à échafauder et qui était au centre de son projet d'organisation d'une Scottish National Convention prévue pour 1939. Gunn, dans une lettre datée juillet 1936, avait ainsi réagi à MacCormick: « The inherent danger of your plan is a subtle dissipation of Nationalist energies and a transference of personal loyalty to another Party. [...] what is now before the Party challenges in a fundamental way the past tactics of the Party. » $(1987$, p. 44) 
14 L'objectif manifeste de MacCormick était de regrouper les partisans nationalistes en une force commune. De nombreuses dissensions internes menaçaient en effet le futur même du SNP, tiraillé entre des membres qui s'identifiaient plus volontiers avec d'autres formations politiques sur l'échiquier politique britannique et européen, certains n'hésitant par exemple pas à se proclamer travaillistes, libéraux ou conservateurs, et d'autres affichant même une attirance non voilée pour les fascistes continentaux. Le manque de cohésion mais aussi l'absence de programme politique cohérent, par exemple sur le plan économique ou sur celui des réponses à donner à la question de l'Empire britannique face à l'auto-détermination écossaise, faisaient perdre toute crédibilité au SNP. À la veille des élections législatives de 1935, le SNP semblait à deux doigts de disparaître en tant que formation politique. Comme le précise Richard Finlay, "[...] the SNP had become more of a movement, rather than a political party. An irreversible decline had begun " (Finlay, 1994, p. 183). Les élections furent par conséquent un désastre et le parti n'en sortit que plus affaibli encore. Un an après, c'est l'extrémiste Andrew Dewar Gibb avec lequel Gunn avait beaucoup échangé de courriers à propos de la fusion des partis nationalistes et la création du SNP, qui confiait ainsi son désarroi dans une lettre à Elma Gibson: "The party seems to be slowly disintegrating " (ibid., p. 189). Toutes les élections partielles qui suivirent furent calamiteuses pour les caciques du parti qui s'y étaient présentés.

15 Highland River, publié deux ans après ces élections, devenait une allégorie sur la nécessité de redéfinir une ligne politique claire. Tous les efforts de médiation que le romancier avait consentis à faire pour la fusion du NPS et du SP avaient été réduits à néant. C'est un retour aux origines de la mouvance nationaliste qui devait se faire. Le nationalisme écossais devait, selon Gunn, se montrer exigeant, sans concession et sans compromis. Dans une lettre inédite adressée à Gibb en septembre 1936, le romancier déclarait : "I can see, for example, a National Party more closely knit than the present body, less tolerant of anything but the pure doctrine, the head and fount of Nationalism, and devoted to propaganda of an undeviating and, if necessary, extreme type ${ }^{1}$." Ce n'est donc pas en tolérant une dispersion des membres vers d'autres partis politiques que l'on pouvait réussir. Il fallait au contraire les rassembler, redéfinir le but du parti et travailler dans un esprit de cohésion. C'est par exemple dans cette même lettre que Gunn explique que le poète Hugh MacDiarmid et ses doctrines marxistes sont une présence incongrue dans tout débat et dans le mouvement politique nationaliste écossais.

16 La symbolique de la remontée jusqu'à la source de la rivière se révèle aussi féconde d'un point de vue littéraire écossais. MacDiarmid et Gunn étaient maintenant clairement en porte-à-faux, non seulement en ce qui concerne la dynamique nationaliste mais aussi à propos du mouvement de la Renaissance. Les conceptions très traditionalistes et nostalgiques de Gunn lui avaient par exemple valu l'opprobre de MacDiarmid. Le poète, sans doute ébranlé par son exclusion du SNP et qui ne pouvait pas ignorer le rôle que Gunn y avait joué, avait accusé ce dernier de colporter le sentimentalisme et le mysticisme celtes. Il était même allé plus loin, et avait dénoncé Gunn comme un mercenaire à la fois de la cause écossaise et de la Renaissance (Gunn, 1987, p. 53-55 et MacDiarmid, 1984, p. 260-261). Là aussi, la remontée de la rivière et le chronotope gunnien prennent une dimension allégorique: les auteurs de la Renaissance devaient, d'après Gunn, revenir aux sources et redécouvrir les fondements mêmes du mouvement, c'est-à-dire donner à l'Écosse une voix spécifique et oublier les différentes écoles littéraires auxquelles ils appartenaient ou prétendaient appartenir, 
et mettre de côté leurs querelles personnelles pour revenir aux fondamentaux de la Renaissance, soit l'expression d'une voix nationale. L'objectif nationaliste, d'abord l'auto-détermination puis l'indépendance, ne devait pas être perdu de vue et c'est autour de lui qu'il fallait se rassembler.

On peut donc dire que Highland River fut un roman clé pour Gunn : l'ouvrage résume bien évidemment la pensée de l'auteur concernant les communautés et les HautesTerres, mais Highland River lui permit en outre de clarifier et de structurer ses figures chronotopiques. Le roman lui offrit également l'occasion d'envisager la politique nationaliste et culturelle de l'Écosse et d'en faire part à ses lecteurs par un biais purement littéraire, celui d'une allégorie.

On ne peut pas ne pas évoquer les chronotopes gunniens et cette allégorie sans les rapprocher de ce que Lewis Grassic Gibbon avait esquissé cinq ans auparavant dans Sunset Song : la persistance de cette terre et le souvenir qu'elle garde des civilisations ou des communautés qui s'y sont succédé. Chris Guthrie, la protagoniste de Gibbon, trouve le réconfort dans le cromlech qui surplombe Kinraddie ; celui-ci lui permet d'échapper à l'oppression patriarcale et au fardeau du travail de la ferme. Gunn fait d'ailleurs une référence très claire à Sunset Song et à ce thème dans la conclusion de son roman, où il parle de «the abiding land» (1937, p. 241), se faisant ainsi l'écho de ce qui devint le célèbre leitmotiv du Quair « the enduring land » (Gibbon, 1932, p. 120).

Des travaux de comparaison entre ces deux romans se révèleraient d'ailleurs fructueux et viendraient compléter ceux qui sont consacrés à Sunset Song et The Silver Darlings (Gifford, 1983). Parmi les pistes qu'il serait possible d'explorer autour de Highland River, on peut dégager des perspectives écologiques et une analyse écocritique des romans de Gunn que l'on pourrait bien sûr lire en parallèle avec la trilogie du Quair de Gibbon. Cela permettrait une analyse de la représentation littéraire de leur paysage où l'homme, et bien évidemment la femme puisque l'on peut parler d'écoféminisme chez Gibbon, apparaissent en complément de leur environnement et y sont perçus en relation symbiotique. Ils investissent ainsi le chronotope bakhtinien de leurs principes idéologiques et de leurs concepts politiques et sociaux.

\section{BIBLIOGRAPHIE}

BAKHTIN Mikhail, 1978, Esthétique et théorie du roman, traduit du russe par Daria Olivier, préface de Michel Aucouturier, Paris, Gallimard.

BAKHTIN Mikhail, 1986, Speech Genres \& Other Late Essays, traduit par Vern W. McGee, Caryl Emerson and Michael Holquist (éds), Austin, University of Texas Press.

CONRAD Joseph, 1990, Heart of Darkness and Other Tales [1899], Introduction de Cedric Watts, Oxford, Oxford University Press.

FinLAY Richard, 1994, Independent and Free. Scottish Politics and the Origins of the Scottish National Party 1918-1945, Édimbourg, John Donald. 
GibBon Lewis Grassic, 1999, Sunset Song [1932], Introduction de Tom Crawford, Édimbourg, Canongate Classics.

GIFFORD Douglas, 1983, Neil M. Gunn and Lewis Grassic Gibbon, Édimboug, Oliver \& Boyd.

GREIMAS Algirdas Julien, 1986, Sémantique structurale. Recherche de méthode [1970], Paris, Presses universitaires de France.

GunN Neil Miller, 1929, « The Hidden Heart » [publié sous le pseudonyme de Dane MacNeil], The Scots Magazine, vol. 9, n 5, p. 331-335.

GunN Neil Miller, 1932, « The Circle », The Scots Magazine, vol. 16, nº 4, p. 241-255.

GunN Neil Miller, 1944, « Exciting Horizons », The Daily Record, 15 août, p. 2.

GuNN Neil Miller, 1952, « Above the Highland Line », National Library of Scotland, DEP 209, box 8.

GunN Neil Miller, 1956, « Caithness », Country Fair, vol. 2, nº 6, p. I-VIII.

GunN Neil Miller, 1961, «Conversation with a Novelist: Maurice Lindsay-Neil Gunn », Scottish Field, $\mathrm{n}^{\circ} 701$, p. 38-39, p. 111.

GunN Neil Miller, 1987, Selected Letters, J.B. Pick (ed.), Édimbourg, Polygon.

GunN Neil Miller, 1991, Highland River [1937], Introduction de Dairmid Gunn, Édimbourg,

Canongate Classics.

HART Francis Russell \& PICK J. B., 1985, Neil M. Gunn: A Highland Life, Édimbourg, Polygon.

LAPLACE Philippe, 2004, «Rites de passage et initiations dans Highland River \& The Silver Darlings de Neil M. Gunn », Études écossaises, n 9, p. 397-410.

LAPLACE Philippe, 2006, Les Hautes-Terres, l'histoire et la mémoire dans les romans de Neil M. Gunn, Besançon, Presses universitaires de Franche-Comté.

LYNCH Peter, 2002, The History of the Scottish National Party, Cardiff, Welsh Academic Press.

MACDIARMID Hugh, 1984, The Letters of Hugh MacDiarmid, Introduction de Alan Bold, Londres, Hamish Hamilton.

Sтокое C. J. L., 1987, A Bibliography of the Works of Neil M. Gunn, Aberdeen, Aberdeen University

Press.

Pour une bibliographie de Gunn mise à jour, voir <www.neilgunn.org.uk>.

\section{NOTES}

1. Pour les échanges de courriers entre Gunn et Gibb et, plus spécifiquement, la dynamique nationaliste, voir : National Library of Scotland, DEP 209, box 15 «Correspondence and printed items concerning the Scottish National Party ». 


\section{RÉSUMÉS}

Cet article examine Highland River, le roman de Neil Gunn publié en 1937 sous un angle bakhtinien en tâchant de reconnaître l'importance que jouent les chronotopes dans le récit. Cet angle de recherche permet en effet d'approcher thématiquement un roman souvent considéré comme de nature difficile à cause de sa structure et des différents genres auxquels il peut être rattaché. Nous verrons ensuite l'utilisation symbolique de la rivière et comment le récit de Highland River s'inscrivent dans une démarche idéologique nationaliste de l'auteur écossais à un moment charnière pour le SNP: le nombre d'adhérents diminuait, d'importants membres exprimaient leurs doutes quant à la ligne politique du parti et il convenait de donner un nouvel élan à la dynamique nationaliste.

The point of this article is to study Neil Gunn's Highland River (1937) under a Bakhtinian light. This allows us to consider the significance of the concept of chronotopes in a novel which has often been regarded as complicated because of its very structure and the various genres it belongs to. We will also examine the symbolic use of the river and how this novel can be considered as being part of Gunn's nationalist ideological discourse at a crucial moment for the future of the SNP: the party's membership was plummeting, key figures were voicing concerns over its political principles, and the nationalist dynamic needed a new boost.

\section{INDEX}

Keywords : Neil Gunn, Highland River, Bakhtin, SNP, Hugh MacDiarmid, Scotland

Mots-clés : Neil Gunn, Highland River, Bakhtine, SNP, Hugh MacDiarmid, Écosse

\section{AUTEUR}

\section{PHILIPPE LAPLACE}

Université de Franche-Comté, laboratoire CRIT.

Philippe Laplace lectures at the Université de Franche-Comté, Besançon. He is the author of a monograph on Gunn, Les Hautes-Terres, l'histoire et la mémoire dans les romans de Neil Gunn (2006) and has edited various books: Cities on the Margins, On the Margins of Cities (2003), Marges et périphéries dans les pays de langue anglaise (2004) and Environmental and Ecological Readings: Nature, Human and Posthuman Dimensions in Scottish Literature and Art (18th-21st c.). 